\title{
Cauda equina compression due to tabetic arthropathy of the spine
}

\author{
P. S. RAMANI AND R. P. SENGUPTA \\ From the Regional Neurological Centre, Newcastle General Hospital, Newcastle upon Tyne
}

SUMMARY A case of tabetic neuroarthropathy of the lumbar spine causing cauda equina compression with paraplegia and retention of urine is described. The literature is reviewed and the role $\nRightarrow$ of surgery in such cases is discussed.

Joint changes in tabetics were first described by Charcot in 1868. However, the German physician Kronig was the first to describe neuroarthropathy of the spine in 1884 (quoted by Alergant, 1960). There are many causes of neuroarthropathy of the spine, tabes dorsalis being the most common. Four to $10 \%$ of tabetics develop Charcot joints and $6-21 \%$ of these involve the spine. The interval between syphilis and arthropathy varies from five to 40 years (McNeel and Ehni, 1969).

The pathology of neurogenic spinal arthropathy is well established (Potts, 1927; Thomas, 1952) and radiological appearances are quite characteristic. The dorso-lumbar and lumbar spine are most commonly affected and more often the lesion is localized to one or two vertebrae so that deformity is localized and acute (Holland, 1952, 1953).

Though the lesion itself is painless, there frequently appears characteristic root pain due to impingement on nerve roots of the proliferative changes in the spine. Root pain when present is often nocturnal, persistent, and distressing (Herndon, 1927). Involvement of nerve roots with resultant sensory disturbances has been described by Campbell and Doyle (1954) and McNeel and Ehni (1969) and several others.

Alergant (1960) claimed to be the first author to report the occurrence of Charcot spine as a cause of motor symptoms and signs due to pressure upon the anterior nerve roots. There are very few cases reported of motor root involvement due to neuroarthropathy of the spine. The role of surgery in syphilis of the spine is therefore not very clear. Few procedures involving surgical decompression of the compromised nerve roots have been described and there is only one report of a case of retention of urine relieved by spinal decompression. In this unit we had the opportunity to treat surgically one case of cauda equina compression, with signs of root com- 근 pression in both lower extremities as well retention of urine due to tabetic neuropathy the lumbar spine.

CASE REPORT

Mr. W.B., a 60 year old retired shoe repairer, was admitted in August 1971 with a four month history of gradual weakness of both lower extremities which $\frac{\mathbb{D}}{\mathbb{D}}$ had been steadily progressive. For one month before $\varrho$ admission he had not been able to walk. He denied $\overrightarrow{\vec{O}}$ any history of lightning pains in the calves or cramps $\exists$ in the legs. He complained of momentary smarting pains around both knee joints for 30 years but these had not stopped. For some two weeks before admission he had been unable to initiate micturition? and had lost bladder sensation.

He fell on his back at the age of 14 years with no serious consequences. A few years later he developed a soft non-tender lump over the lower end of the spine and it was excised under local anaesthesia. We을 do not know the nature of the lump.

At the age of 17 years he had a history of urethral discharge which cleared after one week without 0 treatment. In 1950 at the age of 39 he was treated for $N$ syphilis.

In 1962 when he was 51 he developed pain ando swelling over the right hip, knee, and ankle joints Charcot joints were considered and at that time hec 
underwent some operation on his right hip at some other hospital.

In 1950 his eldest daughter then aged 13 years became gradually blind. She was treated with arsenic and penicillin with gradual recovery of vision at the end of three years. However from the age of 24 years onwards she has been deaf.

The patient was an elderly man with patches of vitiligo over the body and surgical scars over the right hip. The pulse was regular but collapsing and his blood pressure was $190 / 100 \mathrm{~mm} \mathrm{Hg}$. There was an aortic diastolic murmur. His mental state was normal. The pupils were irregular with brown patches in the left iris, with possible minimal direct reaction to light, but a brisk accommodation response. External ocular movements, acuity of vision and fundi were normal and there was no nystagmus. Both upper extremities showed absent biceps and brachioradialis jerks.

In the lower limbs there was moderate wasting of the quadriceps muscle and the right leg was externally rotated. There was generalized weakness of all groups of muscles (grade IV), except plantar flexors where the power was only diminished very slightly. Vibration and joint position sense were absent bilaterally below the iliac crest. Sensation of deep pain was absent. Sensations of touch, pain, and temperature were impaired over the L4, L5, and S1 dermatomes. Plantar reflexes could not be elicited. Both ankle and knee jerks were absent. The bladder was distended up to the umbilicus. There was no tenderness over the spine and no spasm of the paraspinal muscles. The prostate gland was not enlarged.

The blood haemoglobin level was $14.5 \mathrm{~g} / \mathrm{ml}$., ESR

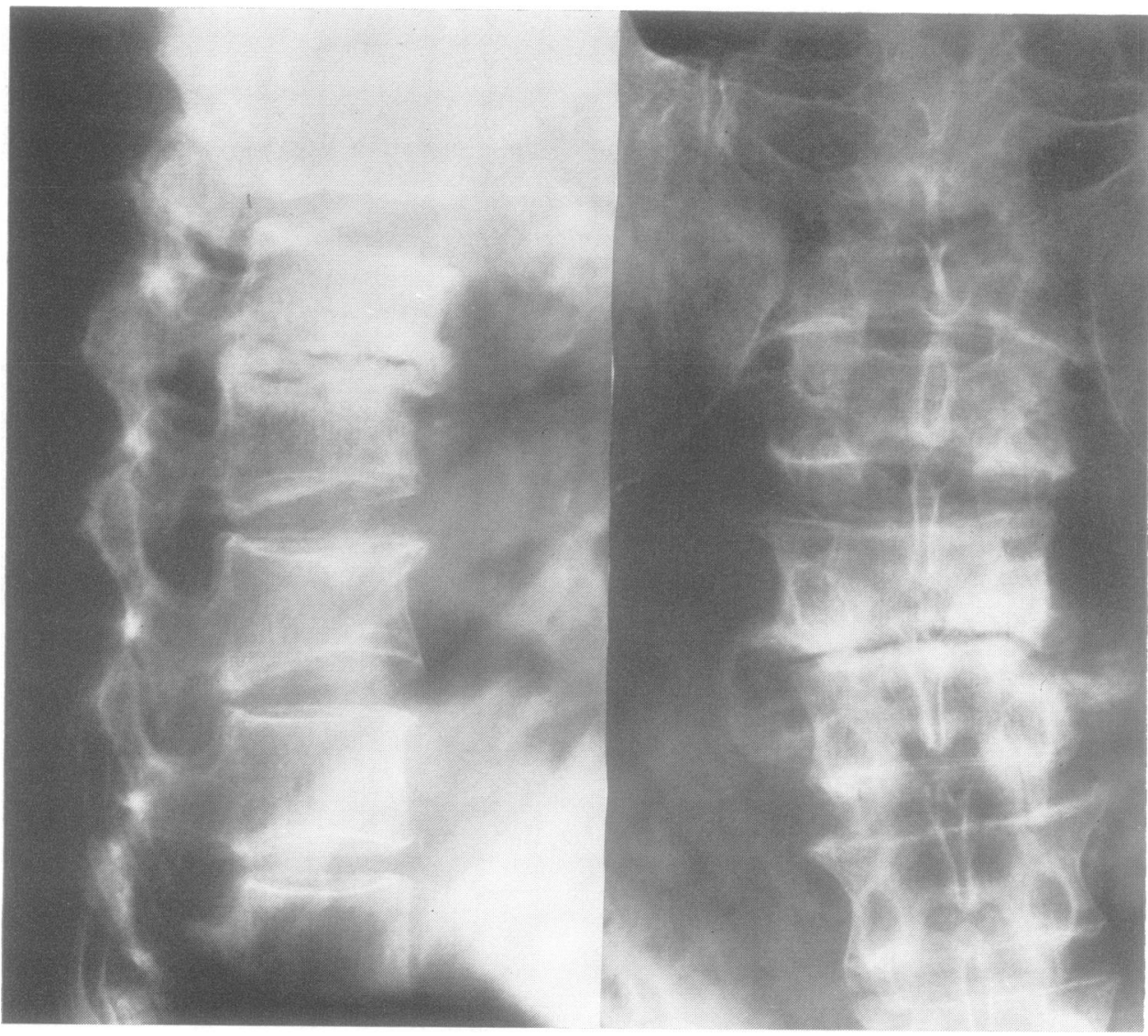

FIG. 1. Anteroposterior and lateral views of the lumbar spine showing gross narrowing of the L3/4 intervertebral disc space with sclerosis of the bodies. There is also some bony debris and marked osteophytic bridging. 


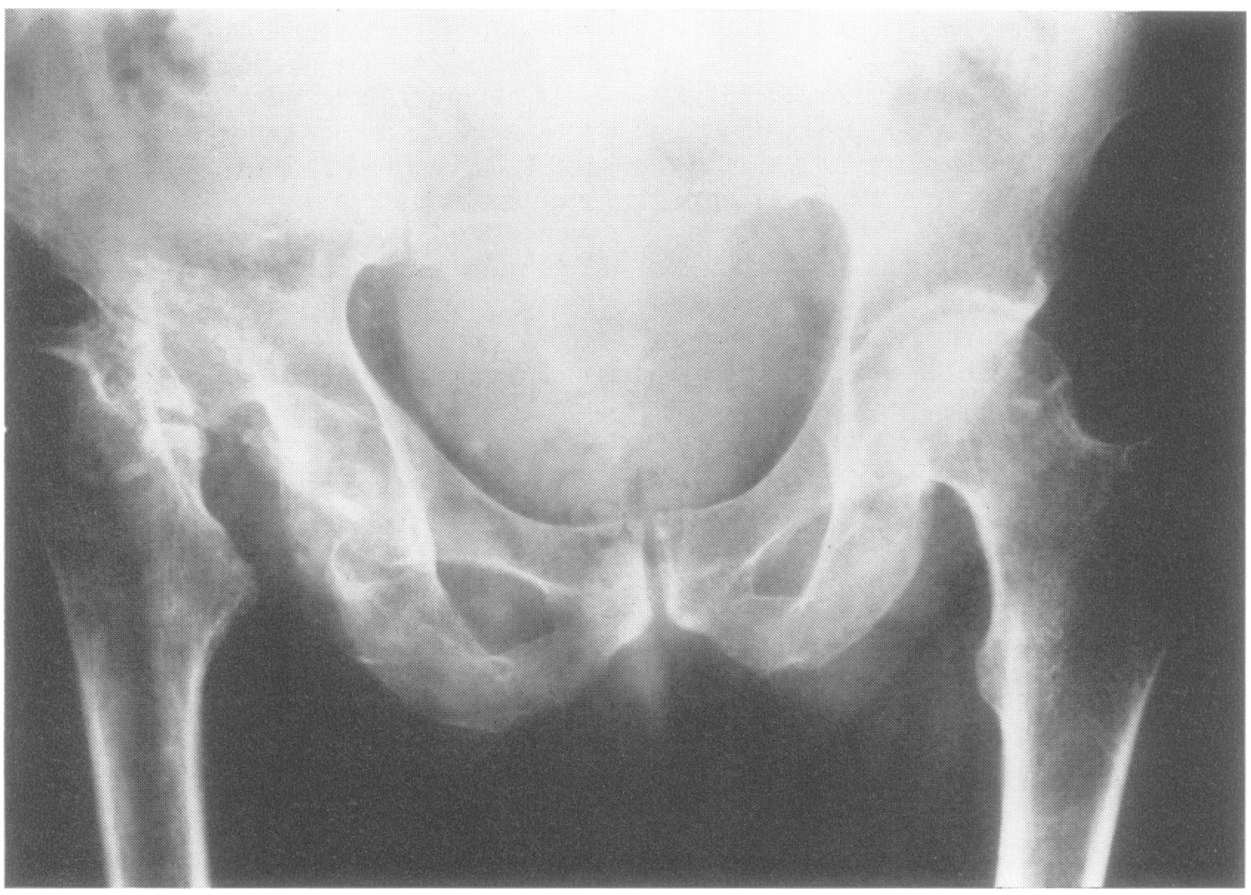

FIG. 2. Complete dislocation and disorganization of the right hip with much bony debris.

FIG. 3. Complete obstruction to the flow of iodophendylate by posterior spondylotic changes (single arrows) and extruded disc material anteriorly (double arrow). 
$48 \mathrm{~mm} / \mathrm{hr}$, fasting blood sugar $82 \mathrm{mg} / 100 \mathrm{ml}$. The blood VDRL reaction was positive. The cerebrospinal fluid (CSF) was clear, colourless, and at normal pressure. It contained $100 \mathrm{mg} / 100 \mathrm{ml}$. protein with $24 \%$ gamma globulins. The VDRL was negative in the CSF. Radiographs of the chest showed marked unfolding of the aorta; radiographs of the cervical spine were normal; while the dorsal spine showed scoliosis. Radiographs of the lumbar spine showed typical features of neuroarthropathy of the spine at L3 and L4 level (Fig. 1). The disc space between L3 and L4 vertebrae was narrowed with changes of degeneration. Bodies of the L3 and L4 vertebrae showed dense sclerosis with formation of large osteophytes. Radiographs of the right hip (Fig. 2) showed neuroarthropathic changes with destruction of the femoral head and formation of a false acetabulum with the ileum. Lumbar route myelography showed complete obstruction to the flow of iodophendylate at the level of L4 vertebra (Fig. 3), with posterior displacement of the column due to degenerated disc material.

$\mathrm{He}$ was started on soluble penicillin $1 \mathrm{Mu}$. daily for three weeks and, in view of the findings at myelography, it was decided to decompress the cauda equina surgically. Total laminectomy of L3 and $\mathrm{L} 4$ vertebrae was carried out, degenerated disc material at the level of L3/L4 was removed, and L4 roots were mobilized on either side. It was also noted that the laminae were very thick and had greatly compromised the space in the spinal canal, tightly compressing the dural sac.

Convalescence was complicated by the development of deep vein thrombosis in the right leg, resulting in a slightly longer stay in the hospital. At the time of discharge, three weeks after the operation, he had regained bladder sensation and was able to void urine normally without hesitancy or incontinence. There was no evidence of dribbling. He was able to walk with support and clinical examination showed considerable improvement in muscular power.

At the end of four and a half months after operation he was able to void urine normally and muscular power in both lower extremities had improved to grade IV.

\section{DISCUSSION}

It is clear that this patient contracted syphilis at the age of 17 years and was admitted 43 years later with clinical evidence of tabes dorsalis and lumbar spinal neuroarthropathy causing nerve root compression in the cauda equina with paraplegia and retention of urine. Surgical decompression gave gratifying results. Within three weeks he had full control over the bladder, paraplegia was gradually improving, and he was up on his feet once again. Loss of vibration and position sense cannot be expected to recover. Alergant (1960) reported two cases with bilateral foot drop. Both cases were treated conservatively. They gradually became worse and ultimately developed wasting of muscles with marked weakness in both lower extremities.

Storey (1954) describing 52 cases of Charcot's joint has recorded one case of spinal Charcot arthropathy with pressure on the lumbosacral plexus giving paraplegia. He has also recorded another patient with collapsed vertebrae due to tabes dorsalis, who developed retention of urine which was relieved by decompression of the spinal canal. No further details are mentioned in either of these cases.

McNeel and Ehni (1969), reporting five cases of neuroarthropathy of the spine, described a patient with possible motor root involvement but who was not treated. Another case had unequivocal evidence of both motor and sensory root involvement and surgical decompression of nerve roots resulted in only temporary relief of symptoms. He required further decompression because of recurrence of symptoms. In view of the rarity of reports on surgical decompression of the nerve roots, it is not possible to draw any firm conclusions as to the treatment of choice. In the past conservative treatment was generally recommended. The efficacy of the fusion of lumbar spine as reported by Storey and others is not very clear.

Many tabetics now enjoy prolonged working life because of penicillin therapy. In the past these patients would have developed progressively incapacitating ataxia or have died from intercurrent infection. Prolongation of working life might mean saving more patients with spinal arthropathy. Surgical decompression of the spinal canal cannot be expected to improve arthropathy. In fact, because of increased mobility, it may tend to hasten the process of destruction of the joints. Decompression might however go a long way to make the life of some of these patients more comfortable and sociable.

We are grateful to Mr. L. P. Lassman and Dr. D. D. Barwick for their permission to publish this case and to the Department of Neuroradiology for kindly supplying the radiographs. 


\section{REFERENCES}

Alergant, C. D. (1960). Tabetic spinal arthropathy. Two cases with motor symptoms due to root compression. British Journal of Venereal Disease, 36, 261-265.

Campbell, D. J., and Doyle, J. O. (1954). Tabetic Charcot's spine: report of eight cases. British Medical Journal, 1, 1018-1020.

Herndon, R. F. (1927). Three cases of tabetic Charcot's spine. Journal of Bone and Joint Surgery, 9, 605-612.

Holland, H. W. (1952). Charcot's arthropathy of the spine, with a note on a case. British Journal of Radiology, 25, 267269.
Holland, H. W. (1953). Tabetic spinal arthropathy. Proceedings of the Royal Society of Medicine, 46, 747-752.

McNeel, D. P., and Ehni, G. (1969). Charcot joint of the lumbar spine. Journal of Neurosurgery, 30, 55-61.

Potts, W. J. (1927). The pathology of Charcot joints. Annals of Surgery, 86, 596-606.

Storey, G. (1964). Charcot joints. British Journal of Venereal Disease, 40, 109-117.

Thomas, D. F. (1952). Vertebral osteoarthropathy of Charcot's disease of the spine. Review of the literature and a report of two cases. Journal of Bone and Joint Surgery, 34B, 248-255. 